\title{
NILPOTENCE AND THE GENERALIZED UNCERTAINTY PRINCIPLE (S)
}

\author{
Nikos Kalogeropoulos \\ Weill Cornell Medical College in Qatar Education City, P.O. Box 24144, Doha, Qatar
}

Received 2014-01-13; Revised 2014-01-13; Accepted 2014-01-23

\begin{abstract}
We point out that some of the proposed generalized/modified uncertainty principles originate from solvable, or nilpotent at appropriate limits, "deformations" of Lie algebras. We briefly comment on formal aspects related to the well-posedness of one of these algebras. We point out a potential relation of such algebras with Classical Mechanics in the spirit of the symplectic non-squeezing theorem. We also point out their relation to a hierarchy of generalized measure theories emerging in a covariant formalism of quantum gravity.
\end{abstract}

Keywords: Uncertainty Principle, Nilpotent/Solvable Groups, Simplistic Topology, Quantum Gravity

\section{INTRODUCTION}

The possibility that the Heisenberg uncertainty principle is modified by quantum gravitational ejects has been rest proposed almost a half century ago (Mead, 1964). More recently, this idea resurfaced (Amati et al., 1989; Konishi et al., 1990; Maggiore, 1993a; 1993b; 1993c; Kempf et al., 1995; Kempf, 1997), mostly motivated by a wish to naturally incorporate a minimal length (Garay, 1995; Jaeckel and Reynaud, 1994) in the various approaches to quantum gravity. Such a generalisation is warranted close to the Planck scale, around which the Compton wavelength of a particle becomes comparable to its Schwarzschild radius. There has been a veritable explosion of interest in this topic during the last two decades, during which formal variations (Das and Vagenas, 2008) and their statistical mechanical and phenomenological implications (Das and Vagenas, 2009; Bambi, 2008; Ali et al., 2009; Bojowald and Kempf, 2012; Chemissany et al., 2011; Majumder, 2012) have been being explored. Implications of the generalized uncertainty principles for quantum eld and gauge theories have also recently emerged (Kim et al., 2009; Kober, 2010; Husain et al., 2012).

It is probably not too surprising that there is no "unique", "natural" or even "best" generalization of the Heisenberg uncertainty principle. Such a generalisation really depends on the goals that one wishes to attain and is ultimately justied by its predictions or by a physical principle, already known or new, that may be uncovered lying at its foundations. As a result, one encounters several versions of the generalised uncertainty principle, which stem from different generalisations of the Heisenberg algebra. Ultimately, such generalised uncertainty principle should arise and be justified by a theory of quantum gravity. Since such a universally acceptable theory is currently lacking several paths, mostly phenomenological motivated, have been taken toward the formulation of generalised uncertainty principles (Amati et al., 1989; Konishi et al., 1990; Maggiore, 1993a; 1993b; 1993c; Kempf et al., 1995; Kempf, 1997; Garay, 1995; Jaeckel and Reynaud, 1994; Das and Vagenas, 2008; 2009; Bambi, 2008; Ali et al., 2009; Bojowald and Kempf, 2012; Chemissany et al., 2011; Majumder, 2012).

In the present work, we examine some formal aspects of such proposed generalisations of the Heisenberg algebra. In section 2, we notice that most such proposed algebras are solvable and even nilpotent in appropriate limits, deformations of Lie algebras. We also comment on why they may fail to have these properties. Such structures interpolate between the Heisenberg algebra, which is 2-step nilpotent, hence solvable and the full structure of a potential non commutative geometry (Chamseddine and Connes, 2010a; 2010b) that may be used to quantize gravity. Some technical points pertaining to some of these algebras are also very briey addressed. In section 3, we make some remarks pertaining to the generalised algebras as seen through the 
"lassical" simplistic non-squeezing theorem (Ekeland and Hofer, 1989; Hofer and Zehnder, 1994; Viterbo, 1989; Arnol'd, 1986; De Gosson, 2001; 2002; 2009a; 2009b; 2012; De Gosson and Luef, 2009; Abbondandolo and Matveyev, 2012) and theirlcounterpart" in the covariant formalism which is a generalized measure theory (Sorkin, 1994; 1997; Salgado, 2002). Section 4, presents an outlook and speculations. In the Appendix we state some very well-known facts from real and Fourier analysis (Stein and Murphy, 1993; Grafakos, 2008; 2009) as well as the theory of pseudo-deferential operators (Taylor, 1991), aiming at making our exposition somewhat smore self-contained.

\subsection{Nilpotence and the Generalized Uncertainty Principle(s)}

The concepts of nilpotent and solvable groups and algebras are central in the structure and classification of both discrete and "continuous" groups and algebras (Helgason, 2001). Let G indicate a group with elements gI where $\mathrm{I}$ is a discrete or continuous index set. The (group) commentator is the subgroup indicated by $[G, G]$ having elements Equation (1):

$$
[\mathrm{G}, \mathrm{G}]=\left\{\mathrm{g}_{\mathrm{i}}^{-1} \mathrm{~g}_{\mathrm{i}}^{-1} \mathrm{~g}_{\mathrm{i}} \mathrm{g}_{\mathrm{j}}, \quad \forall \mathrm{i}, \mathrm{j} \in \mathrm{I}\right\}
$$

In a similar manner, when one considers two subgroups $H_{1}, H_{2} \leq G$, with elements $H_{1}=\left\{h_{1 j}, j \in J\right\} H_{2}=$ $\left\{\mathrm{h}_{2 \mathrm{k}}, \mathrm{k} \in \mathrm{K}\right\}$ with $\mathrm{J}$, $\mathrm{K}$ subsets of $\mathrm{I}$, then their commentator subgroup $\left[\mathrm{H}_{1}, \mathrm{H}_{2}\right]$ is given by Equation (2):

$$
\left[\mathrm{H}_{1}, \mathrm{H}_{2}\right]=\left\{\mathrm{h}_{1 \mathrm{j}}^{-1} \mathrm{~h}_{2 \mathrm{k}}^{-1} \mathrm{~h}_{1 \mathrm{j}} \mathrm{h}_{2 \mathrm{k}} \quad \forall \mathrm{j} \in \mathrm{J}, \mathrm{k} \in \mathrm{K}\right\}
$$

Consider the following commentator groups defined iteratively by Equation (3):

$$
\mathrm{G}_{(1)}=\mathrm{G}, \quad \mathrm{G}_{(\mathrm{i}+1)}=\left[\mathrm{G}, \mathrm{G}_{(\mathrm{i})}\right], \mathrm{i} \in \mathrm{N}
$$

The descending central series is Equation (4):

$$
\mathrm{G} \geq \mathrm{G}_{(2)} \geq \mathrm{G}_{(3)} \geq \ldots
$$

A group $G$ is called n-step nilpotent, if its lower central series terminates after n-steps, namely if there is $\mathrm{n} \in \mathbb{N}$ such that $\mathrm{G}_{(\mathrm{n}+1)}=1$. Several other equivalent dentitions exist for nilpotent groups. Examples of nilpotent groups: All Abelian groups are 1-step nilpotent. The Heisenberg group is 2-step nilpotent. By contrast, the quaternion and the rotation groups are not nilpotent.
Consider the following commentator groups defined iteratively by Equation (5):

$\mathrm{G}^{(1)}=\mathrm{G}, \mathrm{G}^{(\mathrm{i}+1)}=\left[\mathrm{G}^{(\mathrm{i})}, \mathrm{G}^{(\mathrm{i})}\right], \mathrm{i} \in \mathrm{N}$

The derived series is Equation (6):

$\mathrm{G} \geq \mathrm{G}^{(2)} \geq \mathrm{G}^{(3)} \geq \ldots$

A group $\mathrm{G}$ is solvable if its derived series terminates after n' steps, namely if there is $n^{\prime} \in \mathbb{N}$ such that $G^{\left(n^{\prime}+1\right)}=1$. Examples of solvable groups: All Abelian groups are solvable. More generally, all nilpotent groups are solvable, as can be readily seen. A solvable but non-nilpotent group is the symmetric group of 3 elements $S_{3}$. For discrete groups, the Feit-Thompson theorem states that every finite group of odd order is solvable.

For a Lie algebra g similar dentitions apply, by using the matrix instead of the group commentator. Then Engel's theorem states that a Lie algebra is nilpotent if the adjoin $\operatorname{map} \operatorname{ad}_{\mathrm{x}}(\mathrm{y})=[\mathrm{x}, \mathrm{y}], \mathrm{x}, \mathrm{y} \in \mathrm{g}$ is a nilpotent operator, namely if there is $\mathrm{n} \in \mathbb{N}$ such that $\operatorname{ad}(\mathrm{x})^{\mathrm{n}}=0, \forall \mathrm{x} \in \mathrm{g}$. Moreover, $\mathrm{g}$ is solvable if and only if $[\mathrm{g}, \mathrm{g}]$ is nilpotent. The notation for Lie algebras $g$ that we will use is analogous to that for groups, as given above. Analogous dentitions can be used for associative algebras endowed with commentators as will be done in the sequel.

One can easily see that the Heisenberg algebra of Quantum Mechanics, giving rise to the "ordinary" uncertainty principle, is 2-step nilpotent, hence solvable, since Equation (7):

$\left[\mathrm{x}_{\mathrm{i}} \mathrm{p}_{\mathrm{j}}\right]=\mathrm{i} \hbar \delta_{\mathrm{ij}}, \mathrm{i}, \mathrm{j}=1, \ldots, \mathrm{n}$

and all other commutators are zero, where the dimension of the phase space $M$ is $2 n$. Then Equation (8):

$\left[\mathrm{x}_{\mathrm{i}},\left[\mathrm{x}_{\mathrm{j}}, \mathrm{p}_{\mathrm{k}}\right]\right]=\left[\mathrm{p}_{\mathrm{i}},\left[\mathrm{x}_{\mathrm{j}}, \mathrm{p}_{\mathrm{k}}\right]\right]=0$

With the other 2-step commutators trivially zero. Now consider the n-dimensional rotationally symmetric Kempf-Mangano-Mann (KMM) deformation (Kempf et al., 1995; Kempf, 1997) of the Heisenberg algebra given by Equation (9 to 11):

$$
\begin{aligned}
& {\left[\mathrm{x}_{\mathrm{i}}, \mathrm{p}_{\mathrm{j}}\right]=\mathrm{i} \hbar\left(1+\beta^{2}\|\mathrm{p}\|^{2}\right) \delta_{\mathrm{ij}}} \\
& {\left[\mathrm{p}_{\mathrm{i}}, \mathrm{p}_{\mathrm{j}}\right]=0}
\end{aligned}
$$


$\left[\mathrm{x}_{\mathrm{i}} \cdot \mathrm{x}_{\mathrm{j}}\right]=2 \mathrm{i} \hbar \beta^{2}\left(\mathrm{p}_{\mathrm{i}} \mathrm{x}_{\mathrm{j}}-\mathrm{p}_{\mathrm{j}} \mathrm{x}_{\mathrm{i}}\right)$

where, $\beta \in \mathbb{R}_{+}$and Equation (12):

$$
\|\mathrm{p}\|^{2}=\sum_{\mathrm{i}=1}^{\mathrm{n}} \mathrm{p}_{\mathrm{i}} \mathrm{p}_{\mathrm{j}}
$$

We immediately observe that all elements of the second step of the derived series $\mathrm{g}^{(2)}$ are zero, except the following ones that require some straightforward calculations Equation (13 and 14):

$$
\left[\left[\mathrm{x}_{\mathrm{i}} \mathrm{p}_{\mathrm{j}}\right],\left[\mathrm{x}_{\mathrm{k}}, \mathrm{x}_{1}\right]\right]=0
$$

And:

$$
\begin{aligned}
& {\left[\left[\mathrm{x}_{\mathrm{i}}, \mathrm{x}_{\mathrm{j}}\right],\left[\mathrm{x}_{\mathrm{k}}, \mathrm{x}_{1}\right]\right]=2(\mathrm{i} \hbar \beta)^{2}(\mathrm{i} \hbar)\left(1+\beta^{2}\|\mathrm{p}\|^{2}\right)} \\
& \left(\delta_{\mathrm{il}}\left[\mathrm{x}_{\mathrm{j}}, \mathrm{x}_{\mathrm{k}}\right]+\delta_{\mathrm{jk}}\left[\mathrm{x}_{\mathrm{i}} \mathrm{x}_{1}\right]+\delta_{\mathrm{j} 1}\left[\mathrm{x}_{\mathrm{k}} \cdot \mathrm{x}_{\mathrm{i}}\right]+\delta_{\mathrm{ik}}\left[\mathrm{x}_{1}, \mathrm{x}_{\mathrm{j}}\right]\right)
\end{aligned}
$$

Using those, one can express the elements of the higher elements in the derived series in terms of those of $\mathrm{g}$ and $\mathrm{g}^{(2)}$. As can be immediately seen, the derived series does not terminate, in general.

We proceed by further simplifying matters, in order to get armer control of the algebra. An obvious way to achieve this goal is to impose conditions that make $\mathrm{g}^{(2)}$ Abelian. One way to attain this is to consider only the "semi-classical limits" $\hbar \rightarrow 0$ or $\beta \rightarrow 0$, or both, of the KMM deformation. Such a "Inonu-Wigner"-like contraction is implemented by ignoring all terms that are of quadratic or higher order in $\hbar$ and of quartic or higher order in. A second way to proceed is by foregoing altogether all traces of non-commutativity between the "spatial" variables $\mathrm{x}_{\mathrm{i}}$ by imposing Equation (15):

$$
\left[\mathrm{x}_{\mathrm{i}}, \mathrm{x}_{\mathrm{j}}\right]=0
$$

Obviously, (15) is a signicant implication of the KMM deformation. It is adopted by the "modified uncertainty principle" as will be seen in the sequel. If either of these simplications are made, then the corresponding subalgebra of the KMM deformation is 2step solvable as can be seen from (14). We have to be somewhat careful though. If we assume (14) and we omit terms of quadratic and higher order in $\hbar$ then what remains is the Heisenberg algebra, so we get nothing new. Hence, to get a nontrivial result, we are forced, in addition to (14) to omit only terms of quartic or higher order in $\beta$. By using this approximation, we go beyond the Heisenberg algebra, since Equation (16):

$$
\begin{aligned}
& {\left[\left[\mathrm{x}_{1}, \mathrm{x}_{\mathrm{k}}\right],\left[\mathrm{x}_{\mathrm{i}}, \mathrm{p}_{\mathrm{i}}\right]\right]=2(\mathrm{i} \hbar)^{3} \beta^{2}\left(1+\beta^{2}\|\mathrm{p}\|^{2}\right)\left(\delta_{\mathrm{ij}} \delta_{\mathrm{kl}}\right.} \\
& +2(\mathrm{i} \hbar)^{3} \beta^{4} \delta_{\mathrm{ij}}\left\{\left(1+\beta^{2}\|\mathrm{p}\|^{2}\right)\|\mathrm{p}\|^{2} \delta_{\mathrm{kl}}+2\left(1+\beta^{2}\|\mathrm{p}\|^{2}\right) \mathrm{p}_{\mathrm{k}} \mathrm{p}_{1}\right\}
\end{aligned}
$$

Reduces to Equation (17):

$\left[\mathrm{x}_{1},\left[\mathrm{x}_{\mathrm{k}},\left[\mathrm{x}_{\mathrm{i}}, \mathrm{p}_{\mathrm{j}}\right]\right]\right]=2(\mathrm{i} \hbar)^{3} \beta^{2} \delta_{\mathrm{ij}} \delta_{\mathrm{kl}}$

Which is obviously an element of the centre of $g$. Then all 4-step commutators, i.e., all elements of $g_{(5)}$ are trivial. In other words, under the above approximations, the KMM deformation (9)-(11) reduces to a 4-step nilpotent algebra.

Konishi et al. (1990) and Maggiore (1993a; 1993b) proposes a generalization of the Heisenberg uncertainty relations that can be derived from the Lie algebra having generators $x_{i}, p_{j}, J_{k}, i, j, \kappa=1,2,3$ which obey the commutation relations Equation (18 to 23):

$$
\begin{aligned}
& {\left[\mathrm{x}_{\mathrm{i}}, \mathrm{x}_{\mathrm{j}}\right]=-\mathrm{i} \frac{\hbar^{2}}{\kappa^{2}} \in_{\mathrm{ijk}} \mathrm{J}_{\mathrm{k}}} \\
& {\left[\mathrm{x}_{\mathrm{i}}, \mathrm{p}_{\mathrm{j}}\right]=\mathrm{i} \hbar \sqrt{1+\frac{\mathrm{E}^{2}}{\kappa^{2}} \delta_{\mathrm{ij}}}} \\
& {\left[\mathrm{p}_{\mathrm{i}}, \mathrm{p}_{\mathrm{j}}\right]=0} \\
& {\left[\mathrm{~J}_{\mathrm{i}}, \mathrm{x}_{\mathrm{j}}\right]=\mathrm{i} \in_{\mathrm{ijk}} \chi_{\mathrm{k}}} \\
& {\left[\mathrm{J}_{\mathrm{i}}, \mathrm{p}_{\mathrm{j}}\right]=\mathrm{i} \in_{\mathrm{ijk}} \mathrm{p}_{\mathrm{k}}} \\
& {\left[\mathrm{J}_{\mathrm{i}}, \mathrm{J}_{\mathrm{j}}\right]=\mathrm{c}_{\mathrm{ijk}} \mathrm{J}_{\mathrm{k}}}
\end{aligned}
$$

where, $\mathrm{J}_{\mathrm{i}}, \mathrm{i}=1,2,3$ stand for the components of the total angular momentum operator, $\mathrm{c}_{\mathrm{ijk}}$ are the structure constants of the Lie algebra of SU(2) and-is the "deformation" parameter which is identified with the Planck mass. The essential deference between this algebra and the Heisenberg algebra can be essentially traced back to (18). As $\kappa \rightarrow \infty$, we recover the direct product of the Heisenberg algebra with the Lie algebra of SU(2). The latter however cannot become solvable, in any approximation in terms of $\kappa$. To justify this, consider the Killing form of a Lie algebra $g$, defined as the symmetric bilinear form on g given by Equation (24):

$\mathrm{K}(\mathrm{x}, \mathrm{y})=\operatorname{tr}(\operatorname{ad}(\mathrm{x}) \operatorname{ad}(\mathrm{y})), \mathrm{x}, \mathrm{y} \in \mathrm{g}$

Cartan's criterion states that a Lie algebra $g$ is solvable if and only if its Killing form satises Equation (25): 
$\mathrm{K}(\mathrm{x},[\mathrm{y}, \mathrm{z}])=0, \mathrm{x}, \mathrm{y}, \mathrm{z} \in \mathrm{g}$

It is straightforward to check that every subalgebra of a solvable Lie algebra is also solvable. Hence if Maggiore's extension could become solvable in some non-trivial (namely, not resulting in the Heisenberg algebra) approximation in terms of $\kappa$ then its $S U(2)$ subalgebra should also have a degenerate Killing form. This is impossible however as the $\mathrm{SU}(2)$ commutation relations do not depend on the value of $\kappa$ in Maggiore's deformation, as is obvious in (23). Hence Maggiore's deformation cannot give rise to a nilpotent algebra either, in some appropriate limit in terms of $\kappa$. We conclude that our approach and subsequent conclusions do not apply to Maggiore's generalization of the Heisenberg algebra (18)-(23).

The Das-Vagenas (DV) generalised uncertainty relation is a result of the associative algebra endowed with a bracket given by Equation (26 and 27):

$$
\begin{aligned}
& {\left[x_{i}, p_{j}\right]=i \hbar\left(\delta_{i j}+\zeta \delta_{i j}\|p\|^{2}+2 \zeta p_{i} p_{j}\right), i, j=1, \ldots ., n} \\
& {\left[x_{i}, x_{j}\right]=\left[p_{i} p_{j}\right]=0, \quad i, j=1, \ldots ., n}
\end{aligned}
$$

With $\zeta=\zeta_{0} /\left(\mathrm{M}_{\mathrm{Pl}} \mathrm{c}^{2}\right)$ where $\mathrm{M}_{\mathrm{Pl}}$ denotes the Planck mass. This is an anisotropic variation, provided by the term $2 \zeta \mathrm{p}_{\mathrm{i}} \mathrm{p}_{\mathrm{j}}$ of the KMM deformation (9)-(11) with the additional simplification that the spatial coordinates commute as in (15). Requiring (15) instead of (11) is a considerable simplification of the KMM proposal, conceptually more closely aligned to ordinary rather than to non-commutative geometry. As can be readily seen, this algebra is 3-step solvable as all elements of $\mathrm{g}^{(2)}$ are zero. On the other hand, for nilpotency we have Equation (28):

$$
\left[p_{k},\left[p_{i}, p_{j}\right]\right]=0
$$

But Equation (29):

$$
\begin{aligned}
& {\left[\mathrm{x}_{\mathrm{k}},\left[\mathrm{x}_{\mathrm{i}}, \mathrm{p}_{\mathrm{j}}\right]\right]=2(\mathrm{i} \hbar)^{2} \zeta\left(1+3 \zeta\|\mathrm{p}\|^{2}\right) \delta_{\mathrm{ij}} \mathrm{p}_{\mathrm{k}}+2(\mathrm{i} \hbar)^{2}} \\
& \zeta\left\{\left(1+\zeta\|\mathrm{p}\|^{2}\right)\left(\delta_{\mathrm{ik}} \mathrm{p}_{\mathrm{j}}+\delta_{\mathrm{jk}} \mathrm{p}_{\mathrm{i}}\right)+2 \zeta \mathrm{p}_{\mathrm{i}} \mathrm{p}_{\mathrm{j}} \mathrm{p}_{\mathrm{k}}\right\}
\end{aligned}
$$

As was also observed in the case of the KMM algebra, the DV one is not nilpotent unless one resorts to some approximations. The most straightforward assumption is to consider only terms vanishing as quadratic or higher powers of $\hbar$ in which case (29) becomes zero. In this approximation the DV algebra is 3-step nilpotent. On the other hand, someone may wish to keep only terms up to second order in $\zeta$. Then (29) reduces to Equation (30):

$$
\left[\mathrm{x}_{\mathrm{k}},\left[\mathrm{x}_{\mathrm{i}}, \mathrm{p}_{\mathrm{j}}\right]\right]=2(\mathrm{i} \hbar)^{2} \zeta^{2}\left(\delta_{\mathrm{ij}} \mathrm{p}_{\mathrm{k}}+\delta_{\mathrm{ik}} \mathrm{p}_{\mathrm{j}}+\delta_{\mathrm{jk}} \mathrm{p}_{\mathrm{i}}\right)
$$

The only 3-step non-trivial commutator is, in the aforementioned approximation in terms of $\zeta$ Equation (31):

$$
\left[\mathrm{x}_{1},\left[\mathrm{x}_{\mathrm{k}},\left[\mathrm{x}_{\mathrm{i}}, \mathrm{p}_{\mathrm{j}}\right]\right]\right]=2(\mathrm{i} \hbar)^{3}\left(\delta_{\mathrm{ij}} \delta_{\mathrm{kl}}+\delta_{\mathrm{jk}} \delta_{\mathrm{il}}+\delta_{\mathrm{jk}} \delta_{\mathrm{jl}}\right)
$$

Which being a central element of $\mathrm{g}$ implies that Equation (32):

$\left[\mathrm{x}_{\mathrm{m}},\left[\mathrm{x}_{1},\left[\mathrm{x}_{\mathrm{k}},\left[\mathrm{x}_{\mathrm{i}}, \mathrm{p}_{\mathrm{j}}\right]\right]\right]\right]=0$

All the other commutators have been trivially zero from the previous step. We see that the presence of the anisotropic term in (26) does not even aect the step at which the DV algebra becomes nilpotent when compared to the KMM case.

The Ali-Das-Vagenas (ADV) "modied uncertainty principle" (Ali et al., 2009) generalizes the spatialmomentum commutator of the KMM deformation and extends the DV generalized algebra to Equation (33):

$\left[x_{i}, p_{j}\right]=i \hbar\left\{\begin{array}{l}\delta_{i j}-\alpha\left(\|p\| \delta_{i j}+\frac{p_{i} p_{j}}{\|p\|}\right) \\ +\alpha^{2}\left(\|p\|^{2} \delta_{i j}+3 p_{i} p_{j}\right)\end{array}\right\}, i, j=1,2,3$

The generalisation to $\mathrm{n}$ dimensions is straightforward. Here $\alpha=\alpha_{0} 1_{\mathrm{Pl}} / \hbar$ where $1_{\mathrm{Pl}}$ is the Planck length. The ADV algebra assumes, as the DV case (26), (27) above that Equation (34):

$\left[x_{i} x_{j}\right]=\left[p_{i}, p_{j}\right]=0, \quad i, j=1,2,3$

In this case the "deformation" parameter is indicated by $\alpha$. As in the case of (26), (33) is also 3-step solvable since $\mathrm{g}^{(2)}$ is also trivial, as can be seen by a straightforward computation. Notice that due to the fact that the canonical momenta commute (34), the two potentially "dangerous" issues being the exact operator ordering in the fraction of (33), as well as the exact way that $\|\mathrm{p}\|$ and $\frac{1}{\|\mathrm{p}\|}$ are defined, can be temporarily ignored.

To check the nilpotency of the ADV algebra, we will work at a formal level, leaving potential justifications of these steps for Subsections 2.6, 2.7 in the sequel. Consider an operator of interest, let's say $\mathrm{p}_{\mathrm{i}}$. Then define its inverse $\frac{1}{\mathrm{p}_{\mathrm{j}}}$ by demanding Equation (35):

$p_{i} \frac{1}{p_{j}}=\delta_{i j} \quad i, j=1, \ldots, n$ 
There is no need to distinguish, naively at least, a left from a right multiplication, because to due to (34), it is expected that both one-sided multiplications will give the same results. We use repeatedly that the commutator is a derivation, as well as (34) and find from (12) Equation (36):

$$
\left[\mathrm{x}_{\mathrm{k}},\|\mathrm{p}\|^{2}\right]=2 \sum_{\mathrm{j}=1}^{3}\left[\mathrm{x}_{\mathrm{k}}, \mathrm{p}_{1}\right] \mathrm{p}_{1}
$$

With the definition (35) this can be rewritten as Equation (37):

$$
\left[\mathrm{x}_{\mathrm{k}},\|\mathrm{p}\|\right]=\sum_{\mathrm{j}=1}^{3}\left[\mathrm{x}_{\mathrm{k}}, \mathrm{p}_{1}\right] \mathrm{p}_{1} \frac{1}{\|\mathrm{p}\|}
$$

So (35) results in Equation (38):

$$
\left[\mathrm{x}_{\mathrm{k}}, \frac{1}{\|\mathrm{p}\|}\right]=-\left[\mathrm{x}_{\mathrm{k}},\|\mathrm{p}\|\right] \frac{1}{\|\mathrm{p}\|^{2}}
$$

Taking into account (36)-(38), a calculation gives, up to terms of order $\alpha^{2}$, that Equation (39):

$$
\left[\mathrm{x}_{\mathrm{k}},\left[\mathrm{x}_{\mathrm{i}}, \mathrm{p}_{\mathrm{j}}\right]\right]=-(\mathrm{i} \hbar)^{2} \alpha \frac{1}{\|\mathrm{p}\|}\left(\begin{array}{l}
\delta_{\mathrm{ij}} \mathrm{p}_{\mathrm{k}}+\delta_{\mathrm{jk}} \mathrm{p}_{\mathrm{i}}+\delta_{\mathrm{ki}} \mathrm{p}_{\mathrm{j}}-2 \alpha\|\mathrm{p}\| \mathrm{p}_{\mathrm{k}} \delta_{\mathrm{ij}} \\
-\alpha\|\mathrm{p}\| \mathrm{p}_{\mathrm{i}} \delta_{\mathrm{jk}}-\alpha\|\mathrm{p}\| \mathrm{p}_{\mathrm{j}} \delta_{\mathrm{jk}}+\frac{\mathrm{p}_{\mathrm{i}} \mathrm{p}_{\mathrm{j}} \mathrm{p}_{\mathrm{k}}}{\|\mathrm{p}\|^{2}} \\
-4 \alpha \frac{\mathrm{p}_{\mathrm{i}} \mathrm{p}_{\mathrm{j}} \mathrm{p}_{\mathrm{k}}}{\|\mathrm{p}\|^{2}} \\
+\left(\mathrm{i} \hbar^{2}\right) \alpha^{2}\left(2 \mathrm{p}_{\mathrm{k}} \delta_{\mathrm{ij}}+3 \mathrm{p}_{\mathrm{j}} \delta_{\mathrm{ik}}+3 \mathrm{p}_{\mathrm{i}} \delta_{\mathrm{jk}}\right)
\end{array}\right)
$$

We follow the same level of approximation as in the $\mathrm{KMM}$ and DV cases above where terms up to the square of the lowest term in the deformation parameter are retained. Next, we have Equation (40):

$$
\begin{aligned}
& {\left[\mathrm{x}_{1},\left[\mathrm{x}_{\mathrm{k}},\left[\mathrm{x}_{\mathrm{i}}, \mathrm{p}_{\mathrm{j}}\right]\right]\right]=(\mathrm{i} \hbar)^{3} \alpha} \\
& \left\{\begin{array}{l}
-\frac{1}{\|\mathrm{p}\|}\left(\delta_{\mathrm{ij}} \delta_{\mathrm{kl}}+\delta_{\mathrm{jk}} \delta_{\mathrm{il}}+\delta_{\mathrm{ik}} \delta_{\mathrm{jl}}\right) \\
+\frac{1}{\|\mathrm{p}\|^{3}}\left(\delta_{\mathrm{ij}} \mathrm{p}_{\mathrm{k}} \mathrm{p}_{1}+\delta_{\mathrm{jk}} \mathrm{p}_{\mathrm{i}} \mathrm{p}_{1}+\delta_{\mathrm{ik}} \mathrm{p}_{\mathrm{j}} \mathrm{p}_{1}\right. \\
\left.-\delta_{\mathrm{k} \mathrm{l}} \mathrm{p}_{\mathrm{i}} \mathrm{p}_{\mathrm{j}}-\delta_{\mathrm{j} 1} \mathrm{p}_{\mathrm{i}} \mathrm{p}_{\mathrm{k}}-\delta_{\mathrm{il}} \mathrm{p}_{\mathrm{j}} \mathrm{p}_{1}\right) \\
+\frac{\alpha}{\|\mathrm{p}\|^{2}}\left(\begin{array}{l}
5 \delta_{\mathrm{kl}} \mathrm{p}_{\mathrm{i}} \mathrm{p}_{\mathrm{j}}+5 \delta_{\mathrm{j} 1} \mathrm{p}_{\mathrm{i}} \mathrm{p}_{\mathrm{k}}+5 \delta_{\mathrm{il}} \mathrm{p}_{\mathrm{j}} \mathrm{p}_{\mathrm{k}} \\
-\delta_{\mathrm{ij}} \mathrm{p}_{\mathrm{k}} \mathrm{p}_{1}-\delta_{\mathrm{jk}} \mathrm{p}_{\mathrm{i}} \mathrm{p}_{1}-\delta_{\mathrm{ik}} \mathrm{p}_{\mathrm{j}} \mathrm{p}_{1}
\end{array}\right) \\
+\alpha\left(3 \delta_{\mathrm{ij}} \delta_{\mathrm{kl}}+2 \delta_{\mathrm{jk}} \delta_{\mathrm{il}}+2 \delta_{\mathrm{ik}} \delta_{\mathrm{jl}}\right)+3 \frac{\mathrm{p}_{\mathrm{i}} \mathrm{p}_{\mathrm{j}} \mathrm{p}_{\mathrm{k}} \mathrm{p}_{1}}{\|\mathrm{p}\|^{5}} \\
-11 \alpha \frac{\mathrm{p}_{\mathrm{i}} \mathrm{p}_{\mathrm{j}} \mathrm{p}_{\mathrm{k}} \mathrm{p}_{\mathrm{l}}}{\|\mathrm{p}\|^{4}}+(\mathrm{t} \hbar)^{3} \alpha^{2}\left(2 \delta_{\mathrm{ij}} \delta_{\mathrm{k} \mathrm{l}}+3 \delta_{\mathrm{ik}} \delta_{\mathrm{j} 1}+3 \delta_{\mathrm{jk}} \delta_{\mathrm{il}}\right)
\end{array}\right\}
\end{aligned}
$$

Calculation of the next few terms such as $\left[x_{m},\left[x_{1}\right.\right.$, $\left.\left.\left[\mathrm{x}_{\mathrm{k}},\left[\mathrm{x}_{\mathrm{i}}, \mathrm{p}_{\mathrm{j}}\right]\right]\right]\right]$ results in a gradually increasing level of complexity of the resulting expressions, which does not seem to terminate even in the approximation up to $\alpha^{2}$. The reason behind this behavior, which is totally deferent from that of the KMM and the DV algebras, is not hard to pinpoint: It is the existence of $\|\mathrm{p}\|$ rather than of $\|\mathrm{p}\|^{2}$ and its appearance not only in the numerator, but also in the denominator of (33). As long as it is unclear at this point what is the physical principle, if any, dictating the form of (33) and since (33) is not the only expression resulting in an uncertainty relation with desirable phenomenological consequences, it may be prudent to avoid the use of $\|p\|$ itself which introduces these problems for the subsequent formalism. One could use instead integer powers of $\|\mathrm{p}\|$ in any generalized algebra, starting its square as in the KMM (9) or DV (26) cases. Then this algebra will become nilpotent in the lowest non-trivial approximation in terms of the deformation parameter.

In this subsection, we would like to comment on the terms of (33) involving $\|p\|$. It seems that the meaning of this quantity and especially its possible vanishing in the denominator of (33) has not been adequately addressed in the literature of the generalized uncertainty principles, so far. For this reason, a comment or two may be in order about these issues. The notation and some pertinent defintitions used in the rest of this Section, can be found in the Appendix and the references cited therein.

We will assume that someone works in the Schwartz space $S\left(R^{n}\right)$ in which the Fourier transform $F$ is welldefined. Incidentally, it is entirely possible to use another integral transform, such as the Mellin transform, to reach similar conclusions. We immediately see that the symbol corresponding to $\|\mathrm{p}\|$ is Equation (41):

$\tilde{\mathrm{p}}(\mathrm{x}, \mathrm{y})=\left(\mathrm{y}_{1}^{2}+\mathrm{y}_{2}^{2}+\ldots+\mathrm{y}_{\mathrm{n}}^{2}\right)^{\frac{1}{2}}$

This is a classical symbol belonging to the (Hormander) class $S_{1,0}^{1}$. In more physical terms, it is a pure canonical momentum, being independent of the "conjuration" variables $\mathrm{x}$. The corresponding operator $\mathrm{p}\left(\mathrm{x}, \partial_{\mathrm{x}}\right)$ is a rst order pseudo-deferential operator belonging to $\mathrm{OPS}_{1,0}^{1}$. The Laplacian $\nabla^{2}$ on $\mathrm{R}^{\mathrm{n}}$ is a second order elliptic operator, as it has a positive deifnite symbol and we see that (41) can be re-expressed as Equation (42):

$\mathrm{p}\left(\mathrm{x}, \partial_{\mathrm{x}}\right)=\left(\nabla^{2}\right)^{\frac{1}{2}}$ 
As such, $\|\mathrm{p}\|$ is well-defined on $\mathrm{R}^{\mathrm{n}}$. We can re-cast (33) in the slightly different form Equation (43):

$$
\left[x_{i}, p_{j}\right]=i \hbar\left\{\begin{array}{l}
\delta_{i j}-\alpha\|p\|\left(\delta_{i j}+\frac{p_{i}}{\|p\|} \frac{p_{j}}{\|p\|}\right) \\
+\alpha^{2}\|p\|^{2}\left(\delta_{i j}+3 \frac{p_{i}}{\|p\|} \frac{p_{j}}{\|p\|}\right)
\end{array}\right\}, i, j=1,2,3
$$

It should be understood that (33) and (43) are not necessarily equivalent as the domains of these operator expressions can be different, despite their functional equivalence. The interest in operator domains should not be dismissed out of hand as an exercise of purely mathematical interest. Indeed the potential physical importance of operator domains has been investigated for over two decades in the context of quantum gravity and non-commutative geometry, in particular as it pertains to issues of topology change (Asorey et al., 2012; Shapere et al., 2012). We will tacitly assume that the discussion takes place in the intersection of the domains of (33) and (43).

Going back to (43) one should notice that a potential problem lies is its "infra-red" behaviour, namely in the possibility that its denominator becomes zero. This is in stark contrast with typical issues in functional spaces, especially where the Fourier transforms involved, that are mostly concerned about "ultra-violet" divergences. A way to deal with this may be to compactify $\mathbb{R}^{3}$ to the 3torus $\mathrm{T}^{3}$ and only consider functions that obey periodic boundary conditions as is frequently done in Quantum Physics. Another way is to work with principal values of potentially divergent integrals, which is a form of infrared regularization, as is done in the case of Hilbert transforms and other singular integral operators. One can take this path by re-writing the fractional terms of (43) in terms of Riesz transforms as Equation (44):

$$
\left[x_{i}, p_{j}\right]=i \hbar\left\{\begin{array}{c}
\delta_{i j}-\alpha\|p\|\left(\delta_{i j}-R_{i} R_{j}\right) \\
+\alpha^{2}\|p\|^{2}\left(\delta_{i j}-3 R_{i} R_{j}\right)
\end{array}\right\}, i, j=1,2,3
$$

It is well-known (Stein and Murphy, 1993; Grafakos, 2008; 2009) that the Riesz transforms are bounded in $\mathrm{L}^{\mathrm{p}}\left(\mathrm{R}^{\mathrm{n}}\right) ; 1<\mathrm{p}<\infty$. Among them, the $\mathrm{L}^{2}$-integrable functions are of greatest $\beta \in \mathbb{R}_{+}$interest in Physics, hence (43) therefore (33) are well-defined in such spaces, which is sufficient for our purposes.

One issue arising from the ADV algebra (33), (34), due to the presence of the inverse of $\|\mathrm{p}\|$ and its square, is related to smoothness. As can be seen from (12) and (42), in (33) we are dealing with an inverse power of the Laplacian, of the specific form Equation (45):

$$
\mathrm{J}_{\mathrm{s}}=\left(\nabla^{2}\right)-\frac{\mathrm{s}}{2}
$$

For our purposes $s=1$ but it does not hurt to be somewhat more general and allow $s \in \mathbb{C}$, with $\operatorname{Re} s>0$ for convergence purposes. Such expressions are called Riesz potentials of order $\mathrm{s}$ and are defined via the Fourier transform and its inverse, for $f \in 2 S\left(\mathbb{R}^{\mathrm{n}}\right)$ by Equation (46):

$\left(J_{\mathrm{s}} f\right)(\mathrm{x})=\frac{1}{2^{\mathrm{s}} \pi^{\frac{\mathrm{n}}{2}}} \frac{\Gamma\left(\frac{\mathrm{n}-\mathrm{s}}{2}\right)}{\Gamma\left(\frac{\mathrm{s}}{2}\right)} \int_{\mathbb{R n}} \frac{\mathrm{f}(\mathrm{x}-\mathrm{y})}{|\mathrm{y}|^{\mathrm{n}-\mathrm{s}}} \mathrm{d}^{\mathrm{n}} \mathrm{y}$

The effect of operators such as $\mathrm{J}_{\mathrm{s}}$ is to improve the integrability of functions, namely to map $\mathrm{J}_{\mathrm{s}}: \mathrm{L}^{\mathrm{p}} \rightarrow \mathrm{L}^{\mathrm{q}}$ which for $s \in \mathbb{R}$ are related by the Sobolev duality Equation (47):

$\frac{1}{\mathrm{p}}-\frac{1}{\mathrm{q}}=\frac{\mathrm{s}}{\mathrm{n}}$

In the particular case of the ADV algebra above $\mathrm{p}=$ $2, \mathrm{~s}=1, \mathrm{n}=3$ which gives $\mathrm{q}=6$. The Hardy-LittlewoodSobolev fractional integration theorem (Stein and Murphy, 1993; Grafakos, 2008; 2009) provides bounds for the corresponding norms. The Riesz potentials such as $\|\mathrm{p}\|^{-1}$ are essentially integrals, hence they act as smoothing operators. Therefore, by using inverse powers of differential operators, the corresponding expressions become more regular, a clearly desirable property especially for any theory having a classical limit.

On the other hand, because of the fact that integral operators are defined in domains of $\mathrm{R}^{\mathrm{n}}$ rather than points, an obvious question arises about the meaning of locality in models using the ADV algebra. This, does not only create the usual problems of interpretation as in ordinary Quantum Physics but also introduces considerably greater diffculties in the quantization of any such systems. It is not clear, to us at least, what exactly would be the meaning of an operator defined at a point in space, even in the distributional sense, when a non-local operation such as the convolution with a singular integral operator is involved in even defining the algebra expressing the dynamics. A similar issue is also raised and partially addressed in (Maggiore, 1993a) for the KMM algebra by utilising a generalised Bargmann-Fock 
representation and by defining an approximate "quasiposition" representation. One could possibly address such issues by following a largely algebraic path, in the context of generalizations of $C^{*}$ Algebras emulating the path followed in axiomatic quantum eld theory (Haag, 1992) or non-commutative geometry (Chamseddine, 2010a; 2010b). The issue of locality is of central importance in theories of quantum gravity, such as Loop Quantum Gravity (Rovelli, 2011) or Causal Sets (Surya, 2011), which aim to formulate theories that are background independent. Contrast this with the approach taken toward gravity quantization and interaction unification by the String/Brane/M theories (Mukhi, 2011; Blau and Theisen, 2009). Since a generalised uncertainty principle should reflect, in some part, elements of these quantum gravitational theories, its treatment of locality is crucial, extending far beyond the mere technical level that we have alluded to here.

Kempf et al. (1995) and Kempf (1997) an inner product was introduced in an attempt to develop a rudimentary representation aspects of the KMM algebra. It was given, in n-dimensional momentum space, by Equation (48):

$$
(\Psi, \phi)=\int_{\mathbb{R}^{\mathrm{n}}} \frac{1}{1+\beta \mathrm{p}^{2}} \Psi *(\mathrm{p}) \phi(\mathrm{p}) \mathrm{d}^{\mathrm{n}} \mathrm{p}
$$

This product can be seen as "natural" from the viewpoint of the Fourier transform of the inner product of the (Sobolev) Bessel potential space $L_{-1}^{2}\left(R^{n}\right)$. More generally, one can see that the algebras giving rise to the generalised uncertainty principles, can be approximately expressed as "quantizations" in Hilbert spaces endowed with generalized inner products. Modifying the inner product to bypass altogether the Stone-von Neumann theorem, which however does not hold in quantum field theory and obtain distinct predictions from the usual operator quantization of the Fourier modes of the phase space variables is one of the tenets of theories such as Loop Quantum Gravity (Rovelli, 2011). Motivated by the the generalised uncertainty principles as well as by the approach implemented in Loop Quantum Gravity, it may be of some interest to check on whether the Weyl correspondence (Stein and Murphy, 1993) can be modied/extended to apply to the above or any new algebras giving rise to the generalized uncertainty principles.

\subsection{The "Symplectic Camel" and Generalized Measure Theories}

We saw in the previous section that some of the proposed generalisations of the Heisenberg uncertainty principle lead to solvable and in particular limits, to nilpotent Lie algebras. It may be worth wondering on whether this just a coincidence, whether it is part of a mathematical pattern, or even more importantly whether it is a manifestation of a physical principle. This section is an attempt to relate such questions to known facts about Classical Mechanics and a generalized measure hierarchy of potential use for Quantum Gravity, as first rudimentary comments that may contribute toward an answer.

The operator formalism of Quantum Mechanics has undeniable similarities with the Hamiltonian formulation of Classical Mechanics. Then, it would be highly suggestive if an extension of Heisenberg's uncertainty principle could be traced back to the structure of Classical Mechanics. The fact that this is indeed possible for the Heisenberg uncertainty principle itself, is a relatively recently established fundamental result in Symplectic Topology called the "symplectic non-squeezing theorem" (Gromov, 1985; Ekeland and Hofer, 1989; Hofer and Zehnder, 1994; Viterbo, 1989; Arnol'd, 1986; De Gosson, 2001; 2002; 2009a; 2009b; 2012; De Gosson and Luef, 2009; Abbondandolo and Matveyev, 2012) or the principle of the "symplectic camel" (Arnol'd, 1986). As this result has not yet received the visibility in Physics that it duly deserves, despite the extensive eorts of primarily $\mathrm{M}$. De Gosson (and collaborators), who seems to be its biggest advocate in the Physics community (De Gosson, 2001; 2002; 2009a; 2009b; 2012; De Gosson and Luef, 2009), we will say a few words about it that are related to the present work.

The following applies to any symplectic manifold $\mathbf{M}$ but we may wish to think more physically as $M$ being the phase space of a Hamiltonian system. Assume that dim $\mathrm{M}=2 \mathrm{n}$ and let it be parametrized locally by $(\mathrm{x}, \mathrm{p})$ where $\mathrm{x}=\left(\mathrm{x}_{1}, \ldots, \mathrm{x}_{\mathrm{n}}\right)$ and $\mathrm{p}=\left(\mathrm{p}_{1}, \ldots, \mathrm{p}_{\mathrm{n}}\right)$ where the notation is borrowed from the Hamiltonian formulation of Mechanics. Consider the ball Equation (49):

$\mathrm{B}_{2 \mathrm{n}}(\mathrm{R})=\left\{(\mathrm{x}, \mathrm{p}) \in \mathrm{M}:|\mathrm{x}|^{2}+|\mathrm{p}|^{2} \leq \mathrm{R}\right\}$

and the "cylinder" $Z_{1}(r), 1,=1, \ldots, n$ over the symplectic 2-plane $\left(\mathrm{x}_{1}, \mathrm{p}_{\mathrm{l}}\right)$ given by Equation (50):

$$
\mathrm{Z}_{1}(\mathrm{r})=\left\{(\mathrm{x}, \mathrm{p}) \in \mathrm{M}: \mathrm{x}_{1}^{2}+\mathrm{p}_{1}^{2} \leq \mathrm{r}\right\}
$$

Consider a (smooth) canonical transformation (symplectomorphism) f: $\mathrm{M} \rightarrow \mathrm{M}$. The symplectic nonsqueezing theorem states that it is impossible to fit $B_{2 n}$ (R) inside $Z_{l(r)}$ unless $R \leq r$, namely that Equation (51):

$$
\mathrm{f}\left(\mathrm{B}_{2 \mathrm{n}}(\mathrm{R}) \subset \mathrm{Z}_{1}(\mathrm{r}) \Leftrightarrow \mathrm{R} \leq \mathrm{r}\right)
$$


This shows that a phase-space volume is not only preserved by a Hamiltonian (more accurately: A divergence-free) flow, as given by Liouville's theorem, but it possess an additional rigidity associated with its projections along each 2-plane of canonical coordinates. Alternatively, the set of canonical transformations of $\mathrm{M}$ is quite different from the set of volume-preserving transformations of $\mathrm{M}$ (Gromov, 1985; Hofer and Zehnder, 1994; Arnol'd, 1986). This can be interpreted as a rigidity property of Hamiltonian Mechanics whose Quantum Physics "analogue" is the Schrodinger-Robertson inequality (De Gosson and Luef, 2009; De Gosson, 2009a; 2009b; 2012) Equation (52):

$\left(\Delta_{\mathrm{xl}}\right)^{2}\left(\Delta_{\mathrm{pl}}\right)^{2} \geq\left(\operatorname{Cov}\left(\mathrm{x}_{1}, \mathrm{p}_{1}\right)\right)^{2}+\frac{\hbar^{2}}{4}, 1=1, \ldots \mathrm{n}$

where, $\operatorname{Cov}\left(\mathrm{x}_{\mathrm{l}}, \mathrm{p}_{\mathrm{l}}\right)$ stands for an element of the covariance matrix. If the covariance matrix is zero, this results in the usual Heisenberg uncertainty relation. So we see that inside Classical Mechanics itself, there are "elements" of Quantum Physics, when some terms are properly interpreted. Is it possible to use further rigidity results of Classical Mechanics (if any further rigidity exists at all) to guide us in formulating a generalised uncertainty principle, therefore going beyond Quantum Mechanics? This is unclear at present. Although a definitive answer is unknown, it appears that there may be additional rigidity properties in the behaviour of canonical transformations, appearing in the middle dimension $\mathrm{n}$ as the work of (Abbondandolo and Matveyev, 2012) seems to indicate. If such indications are affrmative and more rigidity constraints exist for phasespace volumes, nilpotence in this context would be the termination after a finite number of steps of a sequence of properly defined involutions of such rigidity constraints.

The present work is concerned with nilpotent/solvable associative algebras endowed with a bracket operation, that are non-linear generalisations of Lie algebras and properties of related functional spaces which are the carrier spaces of their representations. It may be worthwhile to see how these ideas may carry over from the canonical to the covariant framework. Each of these two approaches has its own advantages and limitations, but both provide valuable techniques and insights on how to understand and work out the process of quantisation in particular models. As is clear from the generalised uncertainty principles and the corresponding algebras discussed above, our interest is in uncovering properties related to Quantum Gravity.

The most striking observation is that it is not really surprising how different is Quantum Mechanics from
Classical Mechanics, but how actually close they are to each other (Sorkin, 1994; 1997). An indication for such a close relation was provided by the symplectic "nonsqueezing" theorem discussed above. Another is found if one thinks about a triple-slit experiment extending Young's double-slit experiment (Sorkin, 1994; 1997). We start with all three slits open and then gradually start blocking ffo one, then two at a time and then all three. We record the corresponding interference patterns with an overall plus sign if three and one slits are open and with an overall negative sign if two or no slits are open. We superimpose these eight resulting patterns by adding them up algebraically. The result will always be zero. If a four, five slit extension of Young's experiment is set up and calculations are performed along similar lines, the result will always turn out to be zero. This is a direct consequence of the fact that the Heisenberg algebra is 2step nilpotent. In Classical Mechanics no new information beyond the one provided by a "single-slit" experiment is obtained. In Quantum Mechanics, Young's double slit experiment contains all the non-trivial physical information and every multi-slit experiment beyond it gives nothing new. It is in this sense that Quantum Mechanics is as "close" to Classical Mechanics as "possible" (Sorkin, 1994; 1997) although, of course, their structures are quite different from each other.

To generalize this nilpotentcy in the covariant framework, we have to think in terms of generalised measures of histories, expressing the evolution of a system. Consider a set of histories $S_{1}$ having a generalised measure indicated by $\left|S_{1}\right|$. Consider a second set $S_{2}$ and form the disjoint union $\mathrm{S}_{1} \amalg \mathrm{S}_{2}$. These two sets could be chosen to represent the histories of the electron going through slit one or only through slit two in Young's double slit experiment. The extension of the notation and the defintitions to a multislit experiment involving the "histories" $S_{1}, 1=1, \ldots, n$ is immediate. Consider a hierarchy of sum rules (Sorkin, 1994; 1997; Salgado, 2002) Equation (53):

$$
\begin{aligned}
& \mathrm{I}_{1}\left(\mathrm{~S}_{1}\right) \equiv\left|\mathrm{S}_{1}\right| \\
& \mathrm{I}_{2}\left(\mathrm{~S}_{1}, \mathrm{~S}_{2}\right) \equiv\left|\mathrm{S}_{1} \amalg \mathrm{S}_{2}\right|-\left|\mathrm{S}_{1}\right|-\left|\mathrm{S}_{2}\right| \\
& \mathrm{I}_{3}\left(\mathrm{~S}_{1}, \mathrm{~S}_{2}, \mathrm{~S}_{3}\right) \equiv\left|\mathrm{S}_{1} \amalg \mathrm{S}_{2} \amalg \mathrm{S}_{3}\right|-\left|\mathrm{S}_{1} \amalg \mathrm{S}_{2}\right| \\
& -\left|\mathrm{S}_{1} \amalg \mathrm{S}_{3}\right|-\left|\mathrm{S}_{1} \amalg \mathrm{S}_{3}\right|+\left|\mathrm{S}_{1}\right|+\left|\mathrm{S}_{2}\right|+\left|\mathrm{S}_{3}\right| \\
& \mathrm{I}_{\mathrm{n}}\left(\mathrm{S}_{1}, \mathrm{~S}_{2}, \ldots \mathrm{S}_{\mathrm{n}}\right) \equiv\left|\mathrm{S}_{1} \amalg \ldots \mathrm{S}_{\mathrm{n}}\right|-\sum_{\mathrm{l}=1}^{\mathrm{n}}\left|\mathrm{S}_{1} \amalg \ldots \amalg \$ \amalg \ldots \amalg \mathrm{S}_{\mathrm{n}}\right|+ \\
& \sum_{\substack{1_{1}, \mathrm{l}_{2}=\\
\mathrm{l}_{1} \neq \mathrm{I}_{2}}}^{\mathrm{n}}\left|\mathrm{S}_{1} \amalg \ldots \amalg \$_{1_{1}} \amalg \ldots \amalg \mathrm{S}_{1_{2}} \amalg \ldots \amalg \mathrm{S}_{\mathrm{n}}\right|+\ldots+(-1)^{\mathrm{n}+1} \sum_{\mathrm{l}=1}^{\mathrm{n}}\left|\mathrm{S}_{1}\right|
\end{aligned}
$$


Here $\$$ indicates that the argument $\mathrm{S}$ should be omitted in the calculation. Evidently $\mathrm{I}_{1} 6 \neq 0$ for any non-trivial statement to be feasible. Classical Mechanics corresponds to $\mathrm{I}_{2}=0$. Quantum Mechanics is given by $\mathrm{I}_{2} \neq 0, \mathrm{I}_{3}=0$. One can straightforwardly see that $\mathrm{I}_{1+1}=0$ implies that $\mathrm{I}_{1}$ is additive in each of its arguments. This multi-additivity can be used to explain why imposing $\mathrm{I}_{3}=0$ results in being able to express the real part of the decoherence functional as $I_{2}\left(S_{1}, S_{1}\right)=2\left|S_{1}\right|$, which in turn implies that the transition probabilities are proportional to the square of amplitudes, as is well-known in Quantum Physics. A generalized uncertainty principle would refect in this framework that $I_{1} \neq 0,1 \geq 4$. The generalization of the Heisenberg algebra to an l-step nilpotent algebra would be expressed by demanding that $\mathrm{I}_{1+1}=0,1 \geq 4$.

In such theories, the transition probabilities would be functions of some integral power, but not the square, of the amplitudes of wave-functions. It appears that following the covariant approach would also imply that the carrier spaces of the representations of the generalised algebras would be $\mathrm{L}^{\mathrm{p}}\left(\mathbb{R}^{\mathrm{n}}\right), \mathrm{p} \neq 2$, if not more general Sobolev spaces. Such spaces of functions are in general Banach spaces, rather than Hilbert spaces like $L^{2}\left(\mathbb{R}^{n}\right)$ which is the one used Quantum Physics. This poses an obvious problem, as the Banach spaces $\mathrm{L}^{\mathrm{p}}\left(\mathbb{R}^{\mathrm{n}}\right), \mathrm{p} \neq 2$ do not admit an inner product. Then one would have to explain how exactly the geometric structure of the Euclidean spaces stems from that of functions which are elements of $L^{p}\left(\mathbb{R}^{n}\right) p \neq 2$. This might be feasible by technically utilising a Littlewood-Paley type of treatment (Stein and Murphy, 1993; Grafakos, 2008; 2009), but the physical principle that may justify such a "semi/classical" transition $L^{p}\left(\mathbb{R}^{n}\right), p \neq 2$ to $L^{2}\left(\mathbb{R}^{n}\right)$, is not clear to us.

\section{Appendix:}

Here, we collect some very well-known facts from harmonic analysis and pseudo-differential operators that may be of some use in reading Subsections 2.6, 2.7. We follow (Stein and Murphy, 1993; Grafakos, 2008; 2009).

The Schwartz space $S\left(\mathbb{R}^{n}\right)$ is the subspace of smooth functions of $C^{\infty}\left(\mathbb{R}^{n}\right)$ such that themselves as well as their derivatives decay faster than the inverse of any polynomial at infinity. To be more precise, define the multi-indices $\alpha=$ $\left(\alpha_{1}, \ldots \alpha_{n}\right), \beta=\left(\beta_{1}, \ldots \beta_{n}\right)$ with $\alpha, \beta \in \mathbb{N}^{\mathrm{n}}$ by Equation (54):

$x^{\alpha}=x_{1}^{\alpha 1} x_{2}^{\alpha 2} \ldots x_{n}^{\alpha n}, \partial_{x}^{\beta}=\frac{\partial^{\beta 1}}{\partial x_{1}^{\beta 1}} \frac{\partial^{\beta 2}}{\partial x_{2}^{\beta 2}} \ldots \frac{\partial^{\beta n}}{\partial x_{n}^{\beta n}}$

With Equation (55):

$$
|\alpha|=\left|\alpha_{1}\right|+\alpha_{2}|+\ldots+| \alpha_{n}|,| \beta|=| \beta_{1}|+| \beta_{2}|+\ldots .+| \beta_{n} \mid
$$

Consider $\mathrm{f}: \mathbb{R}^{\mathrm{n}} \rightarrow \mathbb{C}$ such that $\mathrm{f} \in \mathrm{C}_{\infty}\left(\mathbb{R}^{\mathrm{n}}\right)$ and for each pair of multi-indices; dene the semi-norms Equation (56):

$\|f\|_{\alpha, \beta}=\sup _{x \in \mathbb{R}^{n}}\left|x^{\alpha} \partial_{x}^{\beta} f(x)\right|$

The above set of denumerable semi-norms allow one to define the Schwartz space by Equation (57):

$\mathrm{S}\left(\mathbb{R}^{\mathrm{n}}\right)=\left\{\mathrm{f} \in \mathrm{C}^{\infty}\left(\mathbb{R}^{\mathrm{n}}\right):\|\mathrm{f}\|_{\alpha, \beta}<\infty, \forall \alpha, \beta \in \mathbb{N}^{\mathrm{n}}\right\}$

One can immediately see that an equivalent definition of $S\left(\mathbb{R}^{n}\right)$, with $C_{\beta N}>0$ constants is Equation (58):

$\left|\partial^{\beta} \mathrm{f}(\mathrm{x})\right| \leq \mathrm{C}_{\beta, \mathrm{N}}(1+|\mathrm{x}|)^{\mathrm{N}}, \forall \beta \in \mathbb{N}^{\mathrm{n}}, \forall \mathrm{N} \in \mathbb{N}$

The dual to $S\left(\mathbb{R}^{\mathrm{n}}\right)$, namely the space of linear functionals on $S\left(\mathbb{R}^{\mathrm{n}}\right)$ is indicated by $S^{\prime}\left(\mathbb{R}^{\mathrm{n}}\right)$ and is called space of tempered distributions of $\mathbb{R}^{\mathrm{n}}$. The Fourier transform for $\mathrm{f} \in \mathrm{S}\left(\mathbb{R}^{\mathrm{n}}\right)$ is defined by Equation (59):

$\mathrm{F}[\mathrm{f}](\xi) \equiv \hat{\mathrm{f}}(\xi)=\frac{1}{(2 \pi)^{\frac{n}{2}}} \int_{\mathbb{R n}} \mathrm{f}(\mathrm{x}) \mathrm{e}^{-\mathrm{ix} \cdot \xi} \mathrm{d}^{\mathrm{n}} \mathrm{x}$

and the inverse Fourier transform is Equation (60):

$$
F^{-1}[f](x) \equiv \hat{f}(x)=\frac{1}{(2 \pi)^{\frac{n}{2}}} \int_{\mathbb{R}^{n}} \hat{f}(\xi) e^{i x . \xi} d^{n} \xi
$$

In the above equations x. $\zeta$ indicates the Euclidean inner product and $|\mathrm{x}|$ stands for the Euclidean norm of $\mathrm{x} \in \mathbb{R}^{\mathrm{n}}$. Both the Fourier and the inverse Fourier transforms are unitary operations (isometries), since according to Parseval's identity Equation (61):

$\int_{\mathbb{R}^{n}} f_{1}(x) f_{2}^{*}(x) d^{n} x=\int_{\mathbb{R}^{n}} \hat{f}_{1}(\xi) \hat{f}_{2}^{*}(\xi) d^{n} \xi$

where, * indicates the complex conjugation and it immediately implies Plancherel's formula Equation (62):

$$
|\mathrm{f}|^{2}=|\hat{\mathrm{f}}|^{2}=|\hat{\mathrm{f}}|^{2}
$$

Consider the function $\tilde{\sigma}(\mathrm{x}, \mathrm{y}): \mathbb{R}^{\mathrm{n}} \times \mathbb{R}^{\mathrm{n}} \rightarrow \mathbb{C}$. For our purposes, it is suffcient to assume that $\sigma \in C^{\infty}\left(\mathbb{R}^{n} \times \mathbb{R}^{n}\right)$. 
Consider $\mathrm{m} \in \mathbb{R}, 0<\mathrm{p}, \delta \leq 1$. Then $\tilde{\sigma}(\mathrm{x}, \mathrm{y})$ is called a symbol in the (Hormander) class $S_{\mathrm{p}, \delta}^{\mathrm{m}}$ if for all multiindices $\alpha, \beta \in \mathbb{N}^{\mathrm{n}}$ there are constants $c_{\mathrm{a}, \beta}$ such that Equation (63 and 64):

$\left|\partial_{x}^{\beta} \partial_{y}^{\alpha} \tilde{\sigma}(\mathrm{x}, \mathrm{y})\right| \leq \mathrm{c}_{\alpha, \beta}\langle\mathrm{y}\rangle^{\mathrm{m}-\mathrm{p}|\alpha|+\delta|\beta|}$

Where:

$$
\langle\mathrm{x}\rangle \equiv\left(1+|\mathrm{x}|^{2}\right)^{\frac{1}{2}}
$$

Consider now the operator $\sigma\left(\mathrm{x}, \partial_{\mathrm{x}}\right): \mathrm{S}\left(\mathbb{R}^{\mathrm{n}}\right) \rightarrow \mathrm{S}\left(\mathbb{R}^{\mathrm{n}}\right)$ given by Equation (65):

$$
\sigma\left(x, \partial_{x}\right) f(x)=\frac{1}{(2 \pi)^{\frac{n}{2}}} \int_{\mathbb{R} n} \tilde{\sigma}(x, \xi) \hat{f}(\xi) e^{-i x \cdot \xi} d^{n} \xi
$$

If $\tilde{\sigma}(\mathrm{x}, \mathrm{y}) \in \mathrm{S}_{\mathrm{p}, \delta}^{\mathrm{m}}$, then $\sigma\left(\mathrm{x}, \partial_{\mathrm{x}}\right)$ is a pseudo-diffierential operator belonging to the class $\mathrm{OPS}_{\mathrm{p}, \delta}^{\mathrm{m}}$. In the above definitions, $\mathrm{m}$ is called the order of the operator. If $\tilde{\partial}$ is polynomial, then the corresponding operator $\sigma$ is differential. If the symbols $\tilde{\partial}(\mathrm{x}, \mathrm{y})$ can be decomposed asymptotically, as sums of homogeneous functions of degrees $m-j$, namely if Equation (66 and 67):

$\tilde{\sigma}(x, y)-\sum_{j=0}^{N} \tilde{\sigma}_{m-j}(x, y) \in S_{1,0}^{\mathrm{m}-\mathrm{N}}$

Where:

$$
\tilde{\sigma}(x, t y)=t^{j} \tilde{\sigma}(x, y), t \in \mathbb{R}|y| \geq 1
$$

then they are called classical symbols. The highest order term in the above classical symbol expansion is called the principal symbol. An element $\sigma \in \mathrm{OPS}_{\mathrm{p}, \delta}^{\mathrm{m}}$ is called elliptic pseudo differential operator, if for some $\mathrm{R}<\infty$ there is a constant $\mathrm{c}>0$ such that Equation (68):

$$
|\tilde{\sigma}(x, y)| \geq c\langle y\rangle^{m},|y| \geq R
$$

Sobolev spaces are spaces of functions aiming to quantify the "degree of the functions' smoothness". First and as a reminder, one defines the Lévesque spaces Equation (69):

$$
\mathrm{L}^{\mathrm{p}}\left(\mathbb{R}^{\mathrm{n}}\right)=\left\{\mathrm{f}: \mathbb{R}^{\mathrm{n}} \rightarrow \mathrm{C}: \int_{\mathbb{R}^{\mathrm{n}}} \mathrm{f}(\mathrm{x}) \mid \mathrm{pd}^{\mathrm{n}} \mathrm{x}<\infty\right\}
$$

It turns out that these are Banach spaces when equipped with the $\mathrm{L}^{\mathrm{p}}$ norm Equation (70):

$\|f\|_{L^{p}}=\left(\int_{R^{n}}|f(x)|^{p} d^{n} x\right)^{\frac{1}{p}}$

For the triangle inequality to hold $1 \leq p \leq \infty$ where $\mathrm{L}^{\infty}$ is equipped with the sup norm. The classical Sobolev spaces $\mathrm{W}^{\mathrm{k}, \mathrm{p}}\left(\mathbb{R}^{\mathrm{n}}\right), \mathrm{k}, \mathrm{p} \in \mathbb{N}$ are defined as Equation (71):

$$
W^{k, p}\left(\mathbb{R}^{n}\right)=\left\{f \in L^{p}\left(\mathbb{R}^{n}\right):\|f\| w^{k, p}=\sum_{|\beta| \leq k}\left\|\partial^{\beta} f\right\| L^{p}<\infty\right\}
$$

An alternative description of $\mathrm{W}^{\mathrm{k}, \mathrm{p}}\left(\mathbb{R}^{\mathrm{n}}\right)$, which also allows for an extension to $\mathrm{k} \in \mathrm{R}$, is given via the Fourier transform and the Bessel potential spaces Equation (72):

$\mathrm{L}_{\mathrm{k}}^{\mathrm{p}}\left(\mathbb{R}^{\mathrm{n}}\right)=\left\{\begin{array}{l}\mathrm{f} \in \mathrm{L}^{\mathrm{p}}\left(\mathbb{R}^{\mathrm{n}}\right): \\ \left\|\mathrm{F}^{-1}\left[\left(1+|\xi|^{2}\right)^{\frac{\mathrm{k}}{2}} \mathrm{~F}[\mathrm{f}(\xi)]\right](\mathrm{x})\right\|_{\mathrm{L}^{\mathrm{p}}}<\infty\end{array}\right\}$

A theorem of Caldero'n states that for $k \in \mathbb{N}$, indeed $\mathrm{W}^{\mathrm{k}, \mathrm{p}}\left(\mathbb{R}^{\mathrm{n}}\right)=\mathrm{L}_{\mathrm{p}}^{\mathrm{k}}\left(\mathbb{R}^{\mathrm{n}}\right)$. Among the above functional spaces, the most commonly used in Physics have, undoubtedly, been $L_{p}^{k}\left(\mathbb{R}^{n}\right)$ and $W^{k, 2}\left(\mathbb{R}^{n}\right)$ both of which are Hilbert spaces. The inner product (...)k of $\mathrm{W}^{\mathrm{k}, 2}\left(\mathbb{R}^{\mathrm{n}}\right)$ is given in terms of the usual $\mathrm{L}^{2}$ inner product (.,.) by Equation (73):

$\left(f_{1}, f_{2}\right) k=\sum_{|\beta| \leq k}\left(\partial^{\beta} f_{1} \partial^{\beta} f_{2}\right)$

Due to the equivalence of the norms $1+|y|$ and $\langle y\rangle$ of $\mathrm{L}^{2}$ one can extend this to an inner product in $\mathrm{W}^{\mathrm{k}, 2}$, $\mathrm{k} \in \mathbb{R}$ by Equation (74):

$\left(\mathrm{f}_{1}, \mathrm{f}_{2}\right) \mathrm{k}=\int_{\mathbb{R}^{\mathrm{n}}}\langle\xi\rangle^{2 \mathrm{k}} \hat{\mathrm{f}}_{1}(\xi) \mathrm{f}_{2}^{*}(\xi) \mathrm{d}^{\mathrm{n}} \xi$

Which gives rise to the norm Equation (75):

$\|\mathrm{f}\|_{\mathrm{k}}^{2}=\int_{\mathbb{R}^{\mathrm{n}}}\langle\xi\rangle^{2 \mathrm{k}}|\hat{\mathrm{f}}(\xi)|^{2} \mathrm{~d}^{\mathrm{n}} \xi$ 
It may be worth observing that if $\mathrm{f} \in \mathrm{S}\left(\mathbb{R}^{\mathrm{n}}\right)$, then $\mathrm{f} \in \mathrm{L}_{\mathrm{p}}^{\mathrm{k}}\left(\mathbb{R}^{\mathrm{n}}\right), \mathrm{k} \in \mathbb{R}$. A pseudo-differential operator, such as $\sigma\left(\mathrm{x}, \partial_{\mathrm{x}}\right) \in \mathrm{OPS}_{\mathrm{p}, \delta}^{\mathrm{m}}$ can be extended to an operator acting between the Sobolev spaces $\mathrm{L}_{\mathrm{k}+\mathrm{m}}^{\mathrm{p}}\left(\mathbb{R}^{\mathrm{n}}\right) \rightarrow \mathrm{L}_{\mathrm{k}}^{\mathrm{p}}\left(\mathbb{R}^{\mathrm{n}}\right)$ or on the space of tempered distributions $S^{\prime}\left(\mathbb{R}^{\mathrm{n}}\right)$.

Riesz transforms are multi-dimensional analogues of the Hilbert transforms. For $\mathbb{R}^{\mathrm{n}}$ the Riesz transforms $\mathrm{R}_{\mathrm{l}}, \mathrm{l}=1, \ldots, \mathrm{n}$ are defined to be singular integral operators of convolution type, as follows: Let $f \in S\left(\mathbb{R}^{n}\right)$. Then Equation (76):

$$
\left(R_{1} f\right)(x)=\frac{\Gamma\left(\frac{n+1}{2}\right)}{\pi \frac{n+1}{2}} \text { p.v. } \int \frac{x_{1}-y_{1}}{|x-y|^{n+1}} f(y) d^{n} y
$$

where, p.v. indicates the principal value of the integral and $\Gamma(\mathrm{x})$ is the Euler gamma function. More explicitly, the Riesz transforms can be seen as the convolutions Equation (77):

$$
\left(R_{1} f\right)(x)=\left(f * \phi_{1}\right)(x)
$$

where, $\phi_{1} \in \mathrm{S}^{\prime}\left(\mathbb{R}^{\mathrm{n}}\right) ; 1=1, \ldots, \mathrm{n}$ are tempered distributions given by the pairing Equation (78):

$$
\left\langle\phi_{1}, \mathrm{~h}\right\rangle=\frac{\Gamma\left(\frac{\mathrm{n}+1}{2}\right)}{\pi^{\frac{\mathrm{n}+1}{2}}} \lim _{\in \rightarrow 0} \int_{|x| \geq \epsilon} \frac{\mathrm{x}_{1}}{|\mathrm{x}|^{\mathrm{n}+1}} \mathrm{~h}(\mathrm{x}) \mathrm{d}^{\mathrm{n}} \mathrm{x}
$$

For $h \in S\left(\mathbb{R}^{n}\right)$. What is of particular interest for our purposes is that the Fourier transform of the Riesz transform is a Fourier multiplier, namely that for $\mathrm{f} \in \mathrm{S}\left(\mathbb{R}^{\mathrm{n}}\right)$, we have Equation (79):

$$
\left(R_{i} f\right)(x)=F^{-1}\left[-i \frac{\xi_{1}}{\xi} F[f](\xi)\right](x)
$$

\section{CONCLUSION}

In this study we attempted to check to what extent some of the, largely, phenomenologicallymotivated generalised uncertainty relations stem from algebras that are solvable, or nilpotent at least in some approximation. We found that if such proposed algebras do not contain a simple part that remains unaffected by the Inonu-Wigner type contraction of one of their deformation parameter (s), then they can be seen as being parts of a solvable algebraic structure. In appropriate limits of parameters depending on the Planck length and mass, such algebras can be seen to possess a nilpotent structure.

It may be worth noticing that the solvable algebras/groups are in a sense complementary to the simple ones that we use extensively in various parts in Classical and Quantum Physics. This complementarily can be seen in two ways: The Killing-Cartan form on solvable Lie algebras is trivial but it is non-zero for simple algebras. Alternatively, any Lie algebra can be expressed as a semi-direct product of a solvable and a semi-simple Lie algebras, according to the LeviMal'tsev decomposition. We are cannot help but wonder on whether this complementarity persists at a more fundamental level and has any significance for Quantum Gravity or it is just a formal coincidence due to our treatment and approximations?

If such a solvability and nilpotency are accepted, then it may be worth examining the form of the generalised measure theories that may be appropriate for formulating the corresponding covariant formalism. In our opinion, this raises obvious questions about the central role that the Hilbert spaces play in Classical and Quantum Physics. We believe that it may be worth further exploring the physical and formal reasons as well as the corresponding implications that may be behind such a role.

The ADV algebra also raises some questions that may be of interest: Should we even allow for pseudodifferential and smoothing operators in fundamental algebras? If so, what may be implications on locality or on the Markovian character of the classical and quantum evolution? What techniques could someone use to explore further such ideas? We believe that some of these questions may merit some attention in future work. Lastly, one cannot fail to see the resemblance of (53) to a simplicial structure. It may be of interest to explore consequences of such a simplical view, define appropriate boundary/coboundary operators and a (co-) homology theory (Spanier, 1994), generalise valuation theory (Klain and Rota, 1997).

\section{ACKNOWLEDGEMENT}

We are grateful to Professor M. De Gosson for bringing his work to our attention and for sending us a copy of (De Gosson, 2009) prior to its publication. 


\section{REFERENCES}

Abbondandolo, A. and S. Matveyev, 2012. How large is the shadow of a symplectic ball. J. Topol. Anal., 5: 87-119.

Ali, A.F., S. Das, E.C. Vagenas, 2009. Discreteness of space from the generalized uncertainty principle. Phys. Lett. B, 678: 497-499. DOI: 10.1016/j.physletb.2009.06.061

Amati, D., M. Ciafaloni and G. Veneziano, 1989. Can spacetime be probed below the string size? Phys. Lett. B, 216: 41-47. DOI: 10.1016/03702693(89)91366-X

Arnol'd, V.I., 1986. First steps in symplectic topology. Russian Math. Surveys. DOI: 10.1070/RM1986v041n06ABEH004221

Asorey, M., A.P. Balachandran, G. Marmo, I.P. Costa e Silva and A.R. de Queiroz et al., 2012. Quantum physics and fluctuating topologies: Survey. Universidad de Zaragoza.

Bambi, C., 2008. Dangerous implications of a minimum length in quantum gravity. Class. Quant. Grav., 25: 105003-105003. DOI: 10.1088/02649381/25/19/195013

Blau, M. and S. Theisen, 2009. String theory as a theory of quantum gravity: A status report. Gen. Rel. Grav., 41: 743-755. DOI: 10.1007/s10714-008-0752-z

Bojowald, M. and A. Kempf, 2012. Generalized uncertainty principles and localization of a particle in discrete space. Phys. Rev., 86: 085017-085017. DOI: 10.1103/PhysRevD.86.085017

Chamseddine, A.H. and A. Connes, 2010a. Space-time from the spectral point of view. American University of Beirut.

Chamseddine, A.H. and A. Connes, $2010 \mathrm{~b}$. Noncommutative geometry as a framework for unification of all fundamental interactions including gravity. Part I. Fortsch. Physik, 58: 553-553. DOI: 10.1002/prop.201000069

Chemissany, W., S. Das, A.F. Ali and E.C. Vagenas, 2011. Effect of the generalized uncertainty principle on post-inflation preheating. JCAP, 12: 017-017. doi:10.1088/1475-7516/2011/12/017

Das, S. and E.C. Vagenas, 2008. Universality of quantum gravity corrections. Phys. Rev. Lett., 101: 221301-221301.

DOI:

10.1103/PhysRevLett.101.221301

Das, S. and E.C. Vagenas, 2009. Phenomenological implications of the generalized uncertainty principle. Can. J. Phys., 87: 233-240. DOI: 10.1139/P08-105
De Gosson, M. and F. Luef, 2009. Symplectic capacities and the geometry of uncertainty: The irruption of symplectic topology in classical and quantum mechanics. Phys. Rep., 484: 131-131. DOI: 10.1016/j.physrep.2009.08.001

De Gosson, M., 2001. The symplectic camel and phase space quantization. J. Phys., 34: 10085-10085. DOI: 10.1088/0305-4470/34/47/313

De Gosson, M., 2002. The 'symplectic camel principle' and semiclassical mechanics. J. Phys., 35: 68256825. DOI: 10.1088/0305-4470/35/32/305

De Gosson, M., 2009a. Symplectic capacities and the geometry of uncertainty: The irruption of symplectic topology in classical and quantum mechanics. Phys. Rep., 484: 131-179. DOI: 10.1016/j.physrep.2009.08.001

De Gosson, M., 2009b. The symplectic camel and the uncertainty principle: The tip of an iceberg? Found. Phys., 39: 194-214. DOI: 10.1007/s10701-0099272-2

De Gosson, M., 2012. The symplectic egg. University of Vienna.

Ekeland, I. and H. Hofer, 1989. Symplectic topology and Hamiltonian dynamics. Math., 200: 355-378. DOI: 10.1007/BF01215653

Garay, L.J., 1995. Quantum gravity and minimum length. Int. J. Mod. Phys., 10: 145-145. DOI: 10.1142/S0217751X95000085

Grafakos, L., 2008. Classical Fourier Analysis. 1st Edn., Springer, Berlin, ISBN-10: 0387094326, pp: 508.

Grafakos, L., 2009. Modern Fourier Analysis. 1st Edn., Springer, New York, ISBN-10: 0387094342, pp: 522.

Gromov, M., 1985. Pseudo holomorphic curves in symplectic manifolds. Inv. Math., 82: 307-347. DOI: 10.1007/BF01388806

Haag, R., 1992. Local Quantum Physics. 2nd Edn., Springer-Verlag, Berlin, ISBN-10: 0387536108, pp: 356.

Helgason, S., 2001. Differential Geometry, Lie Groups and Symmetric Spaces. 1st Edn., American Mathematical Society, ISBN-10: 0821828487, pp: 641.

Hofer, H. and E. Zehnder, 1994. Symplectic Invariants and Hamiltonian Dynamics. 1st Edn., Springer, Basel, ISBN-10: 3764350660, pp: 341.

Husain, V., D. Kothawala and S.S. Seahra, 2012. Generalized uncertainty principles and quantum field theory. Phys. Rev. D, 87: 025014-025018. DOI: 10.1103/PhysRevD.87.025014 
Jaeckel, M.T. and S. Reynaud, 1994. Gravitational quantum limit for length measurements. Phys. Lett., 185: 143-148. DOI: $10.1007 / \mathrm{BF} 01388806$

Kempf, A., 1997. Non-pointlike particles in harmonic oscillators. J. Phys., 30: 2093-2093. DOI: 10.1088/0305-4470/30/6/030

Kempf, A., G. Mangano and R.B. Mann, 1995. Hilbert space representation of the minimal length uncertainty relation. Phys. Rev., 52: 1108-1108. DOI: 10.1103/PhysRevD.52.1108

Kim, Y.W., H.W. Lee and Y.S. Myung, 2009. Thermodynamics of warped AdS3 black hole in the brick wall method. Phys. Lett. B, 673: 90-94. DOI: 10.1016/j.physletb.2009.02.007

Klain, D.A. and G.C. Rota, 1997. Introduction to Geometric Probability. 1st Edn., Cambridge University Press, Cambridge, ISBN-10: 0521596548, pp: 178.

Kober, M., 2010. Gauge theories under incorporation of a generalized uncertainty principle. Phys. Rev., 82: 085017-085017.

DOI: 10.1103/PhysRevD.82.085017

Konishi, K., G. Pauti and P. Provero, 1990. Minimum physical length and the generalized uncertainty principle in string theory. Phys. Lett., 234: 276-284. DOI: 10.1016/0370-2693(90)91927-4

Maggiore, M., 1993a. A generalized uncertainty principle in quantum gravity. Phys. Lett., 304: 6569. DOI: 10.1016/0370-2693(93)91401-8

Maggiore, M., 1993b. Black hole complementarity and the physical origin of the stretched horizon. Phys. Rev., $\quad 49$ : 2918-2921. DOI: 10.1103/PhysRevD.49.2918

Maggiore, M., 1993c. The algebraic structure of the generalized uncertainty principle. Phys. Lett. B, 319: 83-83. DOI: 10.1016/0370-2693(93)90785-G

Majumder, B., 2012. Effects of the modified uncertainty principle on the inflation parameters. Phys. Lett. B, 709: 133-136. DOI: 10.1016/j.physletb.2012.02.022
Mead, C.A., 1964. Possible connection between gravitation and fundamental length. Phys. Rev., 135: B849-B862. DOI: 10.1103/PhysRev.135.B849

Mukhi, S., 2011. String theory: A perspective over the last 25 years. Class. Quant. Grav., 28: 153001153001. DOI: $10.1088 / 0264-9381 / 28 / 15 / 153001$

Rovelli, C., 2011. Thermal time and Tolman-Ehrenfest effect: 'Temperature as the speed of time'. Class. Quant. Grav., 28: 153002-153002. DOI: 10.1088/0264-9381/28/7/075007

Salgado, R.B., 2002. Some identities for the quantum measure and its generalizations. Mod. Phys. Lett., 17: 711-711. DOI: 10.1142/S0217732302007041

Shapere, A.D., F. Wilczek and Z. Xiong, 2012. Models of topology change. University of Kentucky.

Sorkin, R.D., 1994. Quantum mechanics as quantum measure theory. Mod. Phys. Lett., 9: 3119-3119. DOI: 10.1142/S021773239400294X

Sorkin, R.D., 1997. Quantum measure theory and its interpretation. Proceedings of the 4th Drexel Symposium on Quantum Non-integrability: Quantum Classical Correspondence, International Press, Cambridge, Mass.

Spanier, E.H., 1994. Algebraic Topology. 1st Edn., Springer, New York, ISBN-10: 0387944265, pp: 548.

Stein, E.M. and T.S. Murphy, 1993. Harmonic Analysis: Real-variable Methods, Orthogonality and Oscillatory Integrals. 1st Edn., Princeton University Press, Princeton, ISBN-10: 0691032165, pp: 695.

Surya, S., 2011. Directions in causal set quantum gravity. Cornell University Library.

Taylor, M., 1991. Pseudodifferential Operators and Nonlinear PDE. 1st Edn., Springer, Boston, ISBN10: 0817635955, pp: 213.

Viterbo, C., 1989. Symplectic capacities and applications. Asterisque, 177-178: 345. 